\title{
Problématique des plantes envahissantes au sud du Togo (Afrique de l'Ouest) : apport de l'analyse systémique paysagère et de la télédétection
}

\author{
Amah Akodéwou ${ }^{(1,2,4,5)}$, Johan Oszwald ${ }^{(3)}$, Sêmihinva Akpavi ${ }^{(4)}$, Laurent Gazull ${ }^{(1,5)}$, \\ Koffi Akpagana ${ }^{(4)}$, Valéry Gond ${ }^{(1,5)}$ \\ (1) CIRAD, Forêts et Sociétés, 34398 Montpellier, France. E-mail : makodewou@gmail.com \\ (2) AgroParisTech, Université Paris-Saclay, 75732 Paris, France. \\ (3) Université de Rennes, LETG Rennes UMR CNRS 6554, 35043 Rennes, France. \\ (4) LBEV, Université de Lomé, 1515 Lomé, Togo. \\ ${ }^{(5)}$ Forêts et Sociétés, Univ Montpellier, CIRAD, Montpellier, France.
}

Reçu le 13 avril 2018, accepté le 8 janvier 2019, mise en ligne le 7 février 2019.

Cet article est distribué suivant les termes et les conditions de la licence CC-BY (http://creativecommons.org/licenses/by/4.0/ deed.fr)

Description du sujet. Les plantes envahissantes constituent une menace majeure pour les habitats naturels, l'agriculture et la santé dans le monde. Les utilisations du sol sont connues comme étant liées aux invasions végétales. Malgré les mutations d'occupation du sol très rapides et particulièrement autour des aires protégées au Togo, la diversité et l'abondance des plantes envahissantes en lien avec les utilisations du sol sont très peu documentées. Cette étude, par une analyse systémique et la télédétection, se propose d'investiguer le lien entre les utilisations du sol et la diversité des plantes envahissantes dominantes dans et autour de l'Aire Protégée Togodo au sud-est du Togo.

Objectifs. Cette étude poursuit trois objectifs : réaliser une cartographie de l'occupation du sol de la zone d'étude, recenser les principaux éléments paysagers dans et autour de l'Aire Protégée Togodo et leurs moteurs de changement, et inventorier la diversité des plantes envahissantes dominantes dans ces différents éléments paysagers.

Méthode. Une cartographie de l'occupation des sols par approche orientée objet, une typologie des éléments paysagers par une analyse systémique et un inventaire des plantes envahissantes dominantes suivant des transects ont été réalisés.

Résultats. Les pratiques agricoles sont les principaux moteurs des utilisations du sol sur le site. Trente espèces de plantes envahissantes ont été recensées. Il ressort que les jachères sont les plus dominées par les plantes envahissantes.

Conclusions. L'analyse systémique paysagère et la télédétection constituent des outils opérationnels pour une meilleure compréhension de la problématique des plantes envahissantes dans les écosystèmes anthropisés des savanes et forêts sèches de l'Afrique de l'Ouest.

Mots-clés. Chromolaena odorata, Panicum maximum, Spilanthes costata, changement d'utilisation des terres, cartographie de l'occupation du sol, Dahomey Gap.

The issue of invasive plants in Togo (West Africa): contribution of landscape systemic analysis and remote sensing Description of the subject. Invasive plants are a major threat to natural habitats, agriculture and health worldwide. Certain land uses are known to promote plant invasions. Despite the occurrence of rapid land-use mutations, particularly around protected areas in Togo, the diversity and abundance of invasive plants in relation to land use are poorly documented. This prospective study, through systemic analysis and remote sensing, proposed to investigate the land uses and diversity of dominant invasive plants in and around the Togodo Protected Area in southeastern Togo.

Objectives. The objectives of this study were: to map the land use of the study area, to identify both the main landscape elements in and around the Togodo Protected Area and their driving forces of change, and to inventory the diversity of dominant invasive plants within these different landscape elements.

Method. The following were created: a map of land use via an object-oriented approach, a typology of landscape elements through systemic analyses, and an inventory of dominant invasive plants following transects. 
Results. Agricultural practices were found to be the main driving forces behind the land use on the site. Thirty species of invasive plants were identified, and fallows were the areas most dominated by invasive plants.

Conclusions. Systemic landscape analyses and remote sensing are operational tools that can provide a better understanding of the issue of invasive plants within the anthropogenic ecosystems of the savannahs and dry forests of West Africa.

Keywords. Chromolaena odorata, Panicum maximum, Spilanthes costata, landscape dynamics, land cover mapping, Dahomey Gap.

\section{INTRODUCTION}

Une plante envahissante est, d'après Valéry et al. (2008), une plante exotique ou native qui dispose d'un avantage compétitif lui permettant, à la suite de la disparition des obstacles naturels à sa prolifération, de s'étendre rapidement et de dominer de nouvelles aires dans les écosystèmes receveurs, au sein desquels elle devient une population dominante. Les conséquences écologiques, économiques et sociétales des plantes envahissantes sont de plus en plus reconnues à l'échelle planétaire (Pimentel, 2002). La prolifération des plantes envahissantes exotiques constitue l'une des menaces majeures pour la biodiversité au niveau mondial (Elton, 1958 ; Maxwell et al., 2016). En effet, les plantes envahissantes exotiques influencent non seulement la richesse spécifique et la composition, mais aussi modifient les interactions trophiques et les services au sein des écosystèmes (Clavel et al., 2011 ; Vilà et al., 2011). De plus, les plantes envahissantes constituent une menace majeure pour l'agriculture et les moyens de subsistance dans le monde (Paini et al., 2016 ; Pratt et al., 2017). Les changements d'utilisation du sol imposés par les activités humaines sont l'un des principaux facteurs qui facilitent l'établissement, l'abondance et la prolifération des plantes envahissantes (Pyšek et al., 2010 ; Vilà \& Ibáñez, 2011). Aussi, comme l'ont souligné Vilà \& Ibáñez (2011), la vulnérabilité d'un site à être envahi dépend du type d'utilisation du sol, mais aussi des utilisations passées qui ont eu lieu sur ce site. Toutefois, peu de travaux ont cherché à comprendre les plantes envahissantes comme des indicateurs. Pourtant, aborder l'invasion végétale sous cet angle permettrait de saisir le problème en amont et d'éviter de traiter le symptôme plutôt que la cause (Tassin, 2015).

L'éradication des espèces de plantes envahissantes déjà établies sur une vaste zone est rarement possible (Tassin et al., 2007). Les stratégies visant à prévenir les nouvelles introductions et la propagation des plantes envahissantes exotiques sont considérées comme plus efficaces (Leung et al., 2002). Ainsi, comprendre et prédire le succès invasif des plantes est l'un des soucis majeurs de l'écologie des plantes envahissantes. Malheureusement, dans de nombreuses régions du monde, l'ampleur de ce problème n'a pas été suffisamment documentée (Latombe et al., 2017). En dépit des nombreux travaux consacrés aux invasions végétales ces dernières décennies, les lacunes persistantes dans les connaissances scientifiques continuent d'entraver l'aptitude à prédire le succès d'établissement et de prolifération des plantes envahissantes (Bellard \& Jeschke, 2016 ; Pyšek et al., 2017).

Dans les paysages ouest africains, plus de $60 \%$ - et même par endroits plus de $80 \%$ - des habitats naturels auraient été perdus (Burgess et al., 2004). Selon le CILSS (2016), de vastes étendues de savanes et de forêts ont été progressivement remplacées ou fragmentées par les cultures dont les superficies ont doublé entre 1975 et 2013. Ces dynamiques ont favorisé la propagation de nombreuses plantes envahissantes représentant, d'une part, une menace réelle et croissante pour de nombreuses zones de conservation (UICN/PACO, 2013) et, d'autre part, un sérieux problème pour la production agricole (Pratt et al., 2017). À ce jour et à notre connaissance, peu d'études se sont intéressées aux effets des utilisations du sol sur les plantes envahissantes en Afrique de l'Ouest (Aboh et al., 2008). Très peu de recherches ont essayé de bien comprendre l'écologie, la distribution et la gestion des plantes envahissantes en Afrique de l'Ouest (Foxcroft et al., 2010) pour aider les agriculteurs et les gestionnaires des aires de conservation dans leur lutte contre ces plantes (Holou et al., 2013). Pourtant, la compréhension des interactions entre les activités humaines, étudiées à partir des utilisations du sol et la répartition des plantes envahissantes, permettrait de mieux appréhender leur fonctionnement, ce qui faciliterait la mise en place de politiques de gestion plus adéquates. Au Togo, on note dans la plupart des écosystèmes une prolifération des plantes envahissantes. Selon Radji et al. (2010a), la flore togolaise contient 612 espèces introduites, originaires des cinq continents. Beaucoup d'espèces végétales, introduites ou non, constituent de véritables problèmes, envahissant les cultures et les milieux naturels, transformant très nettement la physionomie du paysage, réduisant considérablement les aires de pâturage et conduisant à l'insécurité alimentaire (Akpagana et al., 1993 ; Radji et al., 2010b). D'après ces auteurs, les milieux perturbés tels que les jachères et les forêts dégradées sont respectivement les plus dominées par deux espèces exotiques envahissantes très fréquentes : l'herbe du Laos (Chromolaena odorata [L.] R.M.King \& H.Rob. [Asteraceae]) et le Neem (Azadirachta indica A.Juss. [Meliaceae]). Cependant, 
la diversité et l'abondance des plantes envahissantes en lien avec les dynamiques paysagères sont encore mal connues au Togo malgré les mutations paysagères constatées ces dernières années (Kpedenou et al., 2016), plus particulièrement autour des aires protégées (Fousseni et al., 2012 ; Diwediga et al., 2017).

Cette étude consiste en un état des lieux de la distribution des plantes envahissantes et la dynamique d'occupation/utilisation du sol dans et autour de l'Aire Protégée Togodo (APT). Elle a pour objectif de mettre en évidence l'effet des dynamiques d'occupation/ utilisation du sol sur la distribution et l'abondance des plantes envahissantes. Pour ce faire, elle cherche à répondre aux questions suivantes :

- quelles sont les surfaces et la disposition spatiale des différentes classes d'occupation du sol au sein du milieu ?

- quels sont les principaux éléments paysagers du milieu et comment se transforment-ils ?

- la richesse et la diversité en plantes envahissantes dominantes varient-elles en fonction des éléments paysagers?
Les réponses à ces questions pourront servir à mieux comprendre la répartition spatiale des plantes envahissantes indispensable pour la mise en place des stratégies de gestion plus efficaces.

\section{MATÉRIEL ET MÉTHODE}

\subsection{Site de recherche}

Cette étude a été menée au sud-est du Togo, sur la zone de l'APT et ses alentours immédiats (Figure 1). Le site d'étude, d'une superficie de $1193 \mathrm{~km}^{2}$ et caractérisé par une altitude moyenne de $100 \mathrm{~m}$, se situe à cheval entre le bassin du Mono et le bassin du Haho. Il se trouve dans un ensemble d'habitats et d'écosystèmes uniques en Afrique de l'Ouest, connus sous le nom de Dahomey Gap. Écologiquement, le site se situe dans la plaine côtière ou zone V (Ern, 1979) dominée par les savanes humides guinéennes avec quelques îlots de forêts claires et denses sèches semi-décidues. C'est un site d'une valeur écologique importante renfermant plusieurs zones de conservation de biodiversité tels

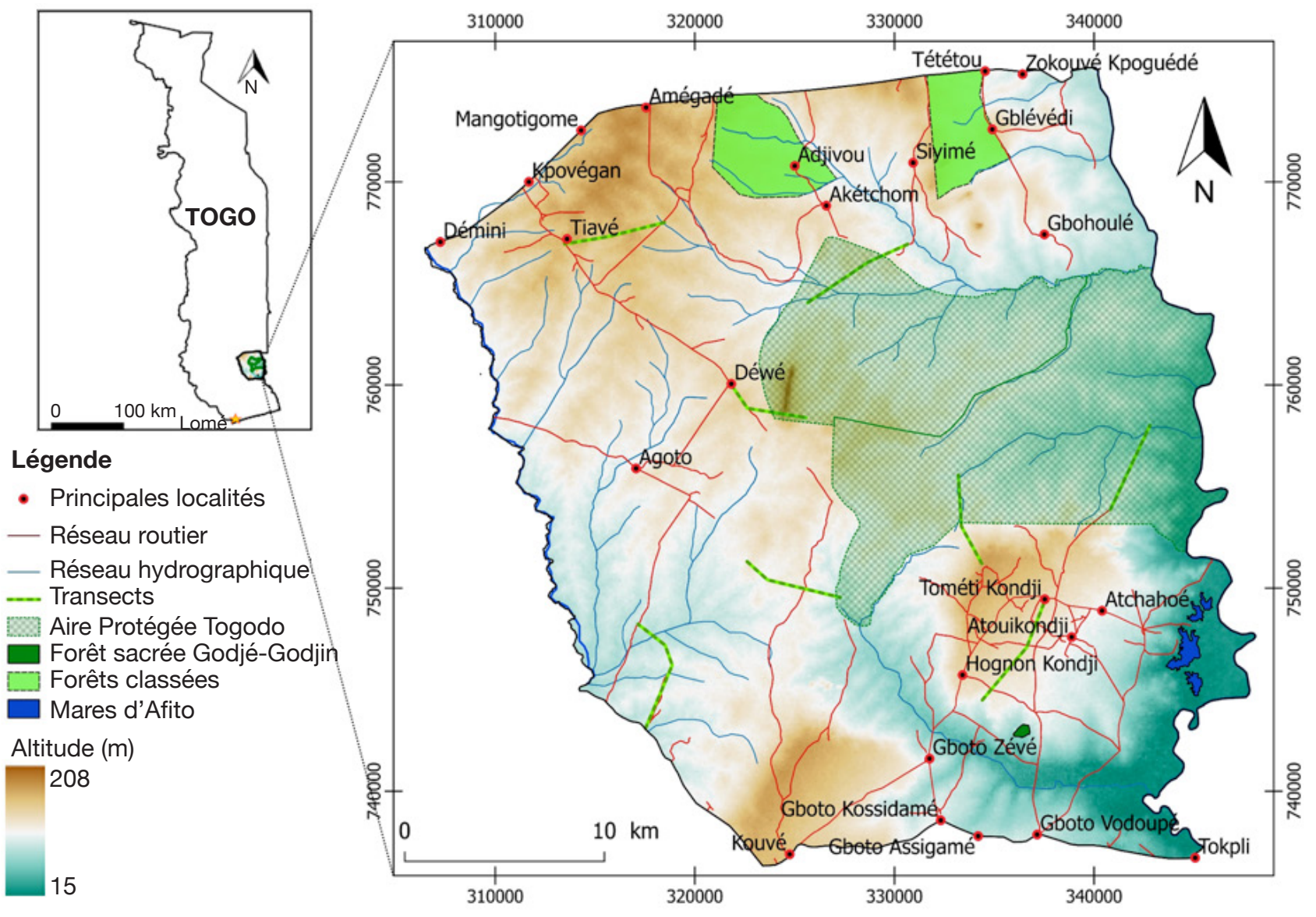

Figure 1. Le site d'étude autour de l'Aire Protégée Togodo, situé au nord-est de Lomé (Togo) - The study site round the Togodo Protected Area, located northeast of Lomé (Togo). 
que l'APT, les forêts classées d'Asrama et de Tététou, la forêt sacrée de Godjé-Godjin et les mares d'Afito qui sont menacées par le développement des activités humaines, notamment agricoles, liées à l'accroissement démographique que connait cette région (DGSCN, 2010). On y retrouve de nombreuses espèces animales vulnérables et en danger sur la liste rouge de l'IUCN telles que l'hippopotame (Hippopotamus amphibius Linnaeus 1758), le singe à ventre rouge qui est une espèce endémique (Cercopithecus erythrogaster erythrogaster Gray, 1866) (Ségniagbéto et al., 2018) et le lamantin d'Afrique (Trichechus senegalensis Link, 1795). Le climat est de type équatorial de transition avec une pluviométrie de 1000 à 1300 mm par an et des températures mensuelles moyennes oscillant entre $25^{\circ} \mathrm{C}$ et $29^{\circ} \mathrm{C}$. La population est essentiellement rurale et se concentre en trois bassins d'implantation avec l'ethnie Adja Ehoué au nord-est, l'ethnie Ewé au nord-ouest et les Ouatchi au sud. L'agriculture est la principale activité développée sur le site. Les activités secondaires exercées sont la carbonisation, l'extraction artisanale de graviers, la chasse, la pêche, la cueillette et le commerce. On trouve sur le site un contraste entre un milieu protégé, avec une végétation semi-naturelle de forêts sèches et de savanes et un milieu fortement anthropisé et hétérogène spatialement parsemé de cultures, de jachères et de villages avec des pratiques agricoles diverses. Du conflit d'usage entre les programmes de conservation et les pratiques de la population locale, il résulte une modification rapide du paysage et de l'agencement des différents écosystèmes. Cela se traduit par une dynamique au niveau de la structure - la taille, la forme, l'arrangement et la distribution des éléments, la composition/diversité des éléments - du paysage et une modification de fonctionnement au sein des communautés végétales progressivement envahies.

\subsection{Cartographie d'occupation des différents éléments paysagers}

La cartographie d'occupation du sol a été réalisée à partir d'une image Sentinel 2 du mois d'avril 2016 (tiles/31/N/CH/2016/4/29/0) téléchargée sur le site de l'ESA Copernicus (https://scihub.copernicus. eu/). Vu la complexité de l'hétérogénéité de notre milieu, l'approche orientée objet a été privilégiée, contrairement à l'approche classique basée sur une classification orientée pixel à partir du spectre. La démarche orientée objet ne traite pas le pixel de manière isolée mais dans son environnement spatial et spectral, en regroupant des pixels au sein d'objets interprétés en se basant sur leurs valeurs spectrales, leur taille, leur forme, leur contexte (Benz et al., 2004). Le logiciel eCognition 9.0 a servi pour notre classification orientée objet. Après l'importation des données prétraitées (corrections géométriques ou radiométriques, géoréférencement) au sein d'un projet, l'image a fait l'objet d'une segmentation et d'une classification. Parmi les différents types d'algorithmes proposés pour la segmentation par le logiciel, nous avons opté pour la segmentation multi-résolution. Cette segmentation autorise d'une part une analyse multi-échelle de l'image, d'autre part l'utilisation de données de source et de nature diverses (Baatz \& Schäpe, 2000). L'approche heuristique par système expert (Robin, 1998) a été retenue pour la classification après segmentation. Le processus de classification est basé sur la formulation d'un certain nombre de règles de connaissance définies par l'utilisateur pour chaque type d'objets à classer (Sparfel et al., 2008). Cette cartographie a servi comme maquette, avec des images Google Earth, pour définir le plan d'échantillonnage et huit transects (Figure 1) de $3 \mathrm{~km}$ à $5 \mathrm{~km}$ de long et de $20 \mathrm{~m}$ de large pour la collecte des points de véritéterrain qui ont servi à l'entrainement et à la validation de la classification (Figure 2). Les campagnes de terrain ont été réalisées de novembre à décembre 2016. Les transects ont été définis suivant l'hétérogénéité du milieu, c'est-à-dire de façon à pouvoir parcourir tous les grands ensembles d'occupation du sol.

Pour l'estimation de la précision de notre classification, nous avons calculé la précision des utilisateurs, la précision des producteurs, la précision globale et l'intervalle de confiance en hectare pour chaque classe d'occupation en suivant l'approche de Olofsson et al. (2013). L'indice de Kappa, généralement utilisé pour l'estimation de la précision des classifications, n'est pas à proprement parler un test et, tout comme un coefficient de corrélation entre deux variables quantitatives, il ne fournit qu'un indicateur numérique de ce que l'on cherche à mesurer, son interprétation reste subjective (Santos, 2018). Selon Pontius \& Millones (2011), les deux estimations de précision des utilisateurs et des producteurs sont beaucoup plus utiles pour résumer une matrice de confusion que les différents indices de Kappa. De plus, étant donné que les estimations de précision sont généralement faites à partir d'un échantillon, ces estimations sont sujettes à des incertitudes qui peuvent être représentées en calculant l'erreur-type ou en rapportant un intervalle de confiance (Olofsson et al., 2013). Dans le cadre de ce travail, nous avons d'abord construit une matrice de confusion à partir des objets (groupe de pixels) sélectionnés par nos 150 points de vérité terrain. Les classes de la classification $(i=1,2$, ...., q) sont représentées par des lignes et les classes de références $(j=1,2, \ldots, q)$ par des colonnes où $q$ est le nombre de classes (7). Cette matrice a servi à construire une seconde matrice de confusion plus objective prenant en compte la proportion de surface de chaque classe (Pontius et al., 2011 ; Olofsson et al., 


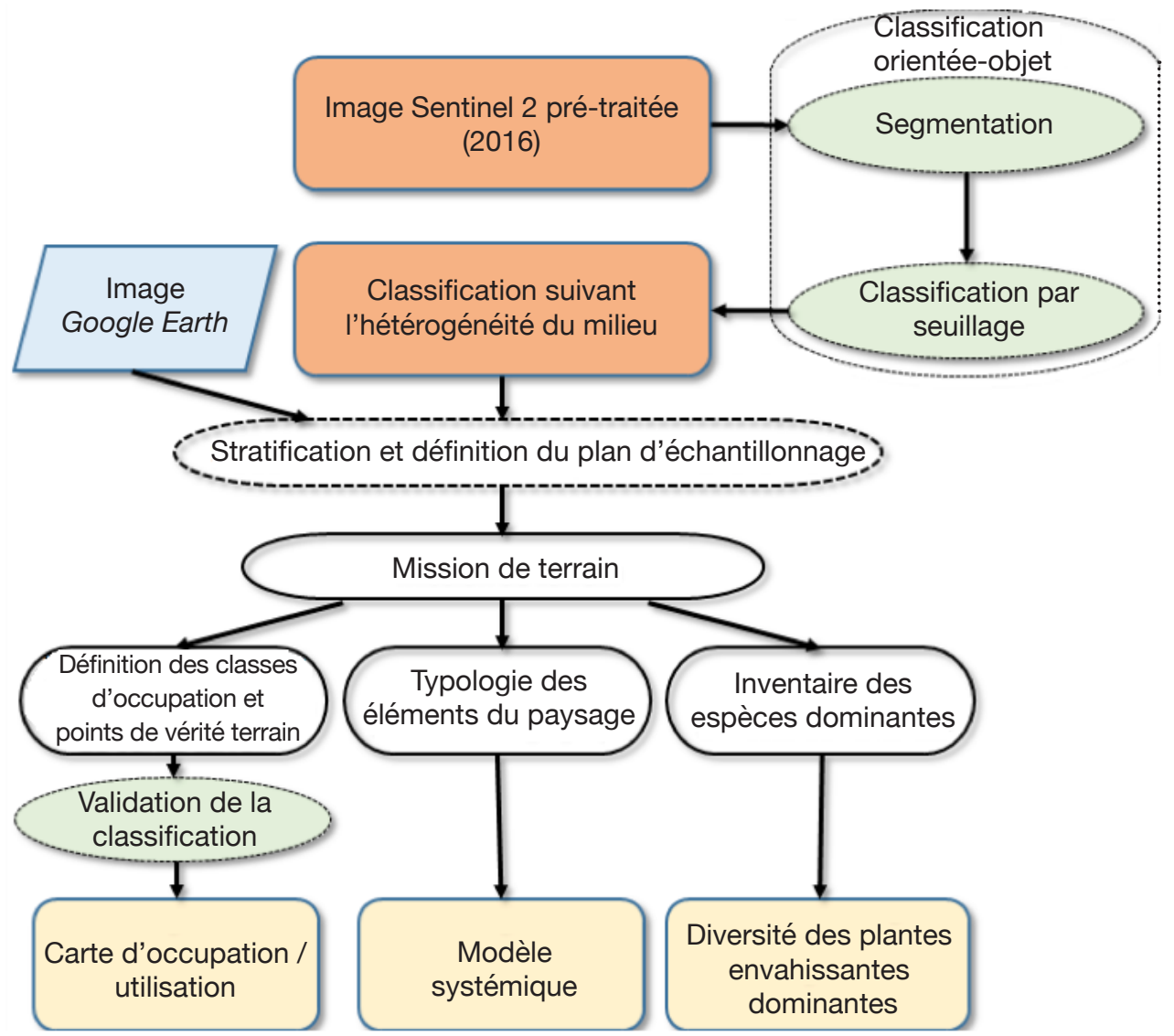

Figure 2. Organigramme de la méthodologie adoptée - Organization chart of the adopted methodology.

2013). La valeur de chaque cellule $i, j$ de la matrice est obtenue par:

$$
\hat{P}_{i j}=W_{i} \frac{n_{i j}}{n_{i}}
$$

où $W_{i}$ est la proportion de surface de la classe $i$, avec

$$
W_{i}=\frac{A_{m, i}}{A_{t o t}}
$$

$A_{m, i}$ étant l'aire de la classe $i$ sur la carte, $A_{t o t}$ étant l'aire totale de la carte, $n_{i j}$ et $n_{i .}$ étant respectivement le nombre de pixels de la cellule $i, j$ et le nombre total de pixels de la classe $i$.

La précision des utilisateurs $U$ et la précision des producteurs $P$ sont calculées respectivement par :

$$
\hat{U}_{i}=\frac{\hat{P}_{i i}}{\hat{P}_{i}}
$$

et

$$
\hat{P}_{j}=\frac{\hat{P}_{j j}}{\hat{P}_{\cdot j}}
$$

avec $P_{i}$ la proportion de surface de la classe de classification $i$ et $P_{, j}$ la proportion de surface de la classe de référence $j$.

La précision globale $O$ de la classification est aussi obtenue à partir de la seconde matrice par :

$$
\hat{O}=\sum_{j=1}^{q} \hat{P}_{j j} .
$$

Ensuite, la surface totale estimée de chaque classe de référence a été calculée par :

$$
\hat{A}_{j}=A_{t o t} \times \hat{P}_{\cdot_{j}}
$$

L'erreur-type d'estimation de la proportion de surface de chaque classe a été déterminée par :

$$
S\left(\hat{P}_{\cdot j}\right)=\sqrt{\sum_{i=1}^{q} W_{i}^{2}} \frac{\frac{n_{i j}}{n_{i} \cdot}\left(1-\frac{n_{i j}}{n_{i} \cdot}\right)}{n_{i} \cdot-1}
$$

De même, l'erreur-type de l'estimation de l'aire de chaque classe est obtenue par :

$$
S\left(\hat{A}_{j}\right)=A_{t o t} \times S\left(\hat{P}_{. j}\right)
$$


Enfin, une approximation de $95 \%$ de l'intervalle de confiance a été déterminée :

$$
\hat{A}_{j} \pm 1,96 \times S\left(\hat{A}_{j}\right)
$$

\subsection{Modèle systémique de dynamiques paysagères}

Afin de mettre en évidence les différentes formes d'emprises ou de conversions anthropiques des terres sur notre site, l'approche systémique des dynamiques paysagères (Oszwald et al., 2007) a été adoptée. Elle consiste, à partir des observations in situ, à établir une typologie des différents écosystèmes - éléments du paysage (Burel \& Baudry, 1999) - de la zone et ensuite à déterminer les interdépendances entre ces éléments. Pour mieux appréhender l'hétérogénéité des mosaïques très complexes de notre site, une stratification a été appliquée (Chatelain et al., 1996). Ainsi, des grands ensembles paysagers ont été identifiés à partir d'une pré-stratification basée sur l'interprétation d'images issues de Google Earth et d'une cartographie d'occupation du sol de l'année 2016 (Figure 2). Au niveau de ces grands ensembles paysagers, nous avons recensé les différents écosystèmes ou éléments du paysage le long des transects. Plusieurs informations ont été renseignées au niveau du centre de chaque élément paysager: les coordonnées géographiques, la physionomie générale et une série de quatre photos orientées (Nord, Est, Sud et Ouest) afin de garder en mémoire la nature de la zone où la mesure a été effectuée. Au fur et à mesure des constatations, une typologie a été mise en place (Oszwald et al., 2010). De plus, quelques entretiens informels avec des paysans rencontrés lors de nos relevés ont permis d'améliorer notre compréhension sur les pratiques culturales, les types de changements d'occupation/utilisation du sol et les facteurs qui sous-tendent les changements.

\subsection{Diversité des plantes envahissantes dominantes}

Dans le but de déterminer la diversité et la fréquence d'occurrence des plantes envahissantes dominantes, des relevés floristiques ont été réalisés le long des huit transects dans des placettes de $50 \mathrm{~m}$ x $50 \mathrm{~m}$ installées de façon aléatoire au milieu des différents écosystèmes rencontrés. Au niveau de chaque placette, les espèces « dominantes » (les espèces ayant un coefficient 4 ou 5 suivant l'échelle d'abondance-dominance de BraunBlanquet) ont été notées en présence/absence. Cette échelle (Braun-Blanquet et al., 1952) comprend six niveaux : + (individus rares ou très rares et recouvrement très faible); 1 (individus assez abondants, mais recouvrement faible); 2 (individus très abondants, recouvrement au moins $1 / 20$ ) ; 3 (nombre d'individus quelconque, recouvrement $1 / 4$ à 1/2); 4 (nombre d'individus quelconque, recouvrement $1 / 2$ à $3 / 4$ ) et 5 (nombre d'individus quelconque, recouvrement plus de 3/4). La définition d'espèce «dominante» retenue dans notre étude a été héritée de Dessaint et al. (2001). Les espèces végétales dont l'identification était confuse sur le terrain ont été photographiées ou échantillonnées afin d'assurer leur identification au Laboratoire de Botanique et Écologie Végétale de l'Université de Lomé (Togo). La nomenclature de The Plant List (2013) a servi à nommer les taxons. Le statut envahissant des espèces exotiques a été défini sur la base des listes provenant d'études : sur les espèces des plantes envahissantes du monde (Weber, 2003), sur les plantes exotiques envahissantes des savanes tropicales et subtropicales (Foxcroft et al., 2010), de Global Invasive Species Database (UICN, 2017) et de Invasive Species Compendium (CABI, 2017). Pour les espèces natives, le statut a été défini sur la base de leur agressivité - avantage compétitif des espèces sur les autres leur permettant de dominer et de former des peuplements presque monospécifiques (Valéry et al., 2008) - observée sur le terrain.

La richesse spécifique totale et par occupation du sol en plantes envahissantes dominantes a été déterminée par le nombre total d'espèces envahissantes dominantes observées dans l'ensemble des relevés. La fréquence relative d'occurrence de chaque espèce envahissante dominante a été calculée par $\mathrm{Fr}=n i / N$, où $n i$ : nombre de relevés dans lequel l'espèce $i$ est présente (fréquence absolue) et $N$ le nombre total de relevés. Trois classes ont été définies: espèces préférentielles ou très fréquemment présentes $(\mathrm{Fr}>20 \%)$; espèces rarement présentes $(5<\mathrm{Fr} \leq 20 \%)$ et espèces très rarement présentes $(\mathrm{Fr} \leq 5 \%)$ dans les éléments paysagers du site d'étude. Nous avons comparé la diversité en plantes envahissantes dominantes des différentes occupations du sol à l'aide de courbes de raréfaction et d'extrapolation (Colwell et al., 2012) en fonction du nombre de relevés grâce au logiciel EstimateS 9.1.0. Ensuite, un test de Khi carré a été réalisé pour évaluer l'indépendance entre les classes d'occupation du sol et la richesse en plantes envahissantes dominantes. Enfin, nous avons évalué la liaison entre les espèces de plantes envahissantes dominantes et les classes d'occupation du sol à l'aide d'un diagramme en mosaïque avec des résidus standardisés selon un modèle log-linéaire (Friendly, 1994).

\section{RÉSULTATS}

\subsection{Cartographie d'occupation/utilisation du milieu pour 2016}

Les résultats de l'évaluation de la précision de classification sont présentés dans les tableaux 1, 2 et 
3. La précision globale de notre cartographie est de $88 \%$ (Tableau 3). Concernant l'organisation spatiale des différentes occupations/utilisations du sol, on retrouve un contraste net entre les forêts et les savanes situées principalement à l'intérieur de l'aire protégée et les terres cultivées (champs, jachères et plantations) à l'extérieur (Figure 3).

Plus des 3/4 (78\%) de la superficie de la zone d'étude sont occupés par les bâtis, les champs, les plantations et les jachères. Les espaces semi-naturels que sont les forêts et les savanes ne représentent respectivement qu'environ $6 \%$ et $16 \%$, soit $7609 \pm$ 1053 ha et $19283 \pm 1850$ ha selon les estimations et respectivement 6994 ha et 19696 ha sur la carte (Tableaux 1 et 2).

\subsection{Modèle systémique des dynamiques d'occupation/utilisation}

Le modèle systémique des dynamiques d'occupation/ utilisation du sol fournit des informations sur les types d'occupation/utilisation et les interdépendances entre ces éléments.

On distingue sur notre site deux grands types d'écosystèmes ou d'éléments paysagers en fonction du degré de perturbation par l'homme :

- les éléments paysagers fortement perturbés: les cultures (monoculture ou cultures associées), les jachères, les plantations de palmier à huile (Elaeis guineensis Jacq.) ou de teck (Tectona grandis L.f.) et les recrues forestières ;

- les éléments paysagers peu perturbés : principalement situés à l'intérieur de l'aire protégée, on y distingue les savanes (arbustives, arborées et boisées) et les forêts (claires, sèches semi-décidues et galeries) (Figure 4).

\section{Les éléments paysagers fortement perturbés}

Les cultures. L'agriculture sur brûlis est le système le plus utilisé dans la zone. Après le défrichement par abatis-brûlis des forêts et/ou savanes ou rarement des vieilles jachères et plantations (flèche rouge), les champs sont installés soit en monoculture ou en cultures associées. Les monocultures sont surtout le maïs (Zea mays L.), l'igname (Dioscorea alata L.), le coton (Gossypium hirsutum L.) et le riz (Oryza sativa L.). Les associations de cultures se font souvent entre le maïs, le manioc (Manihot esculenta Crantz), le niébé (Vigna unguiculata [L.] Walp.), l'arachide (Arachis hypogaea L.), la tomate (Solanum lycopersicum L.), le piment (Capsicum annuum L.), le palmier à huile (E.guineensis) et le teck (T.grandis). Les labours à plats, en butte ou en sillon sont les types les plus pratiqués. Les cultures sur bas-fonds sont dominées par les adventices telles que Spilanthes costata Benth., Ageratum conyzoides (L.) L. et Blumea viscosa (Mill.)

Akodéwou A., Oszwald J., Akpavi S. et al.

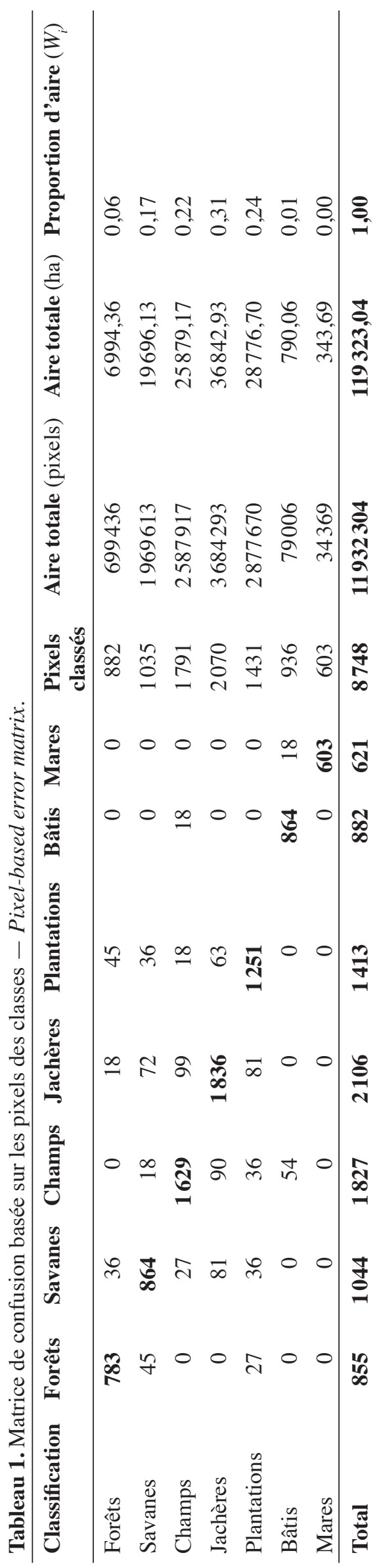



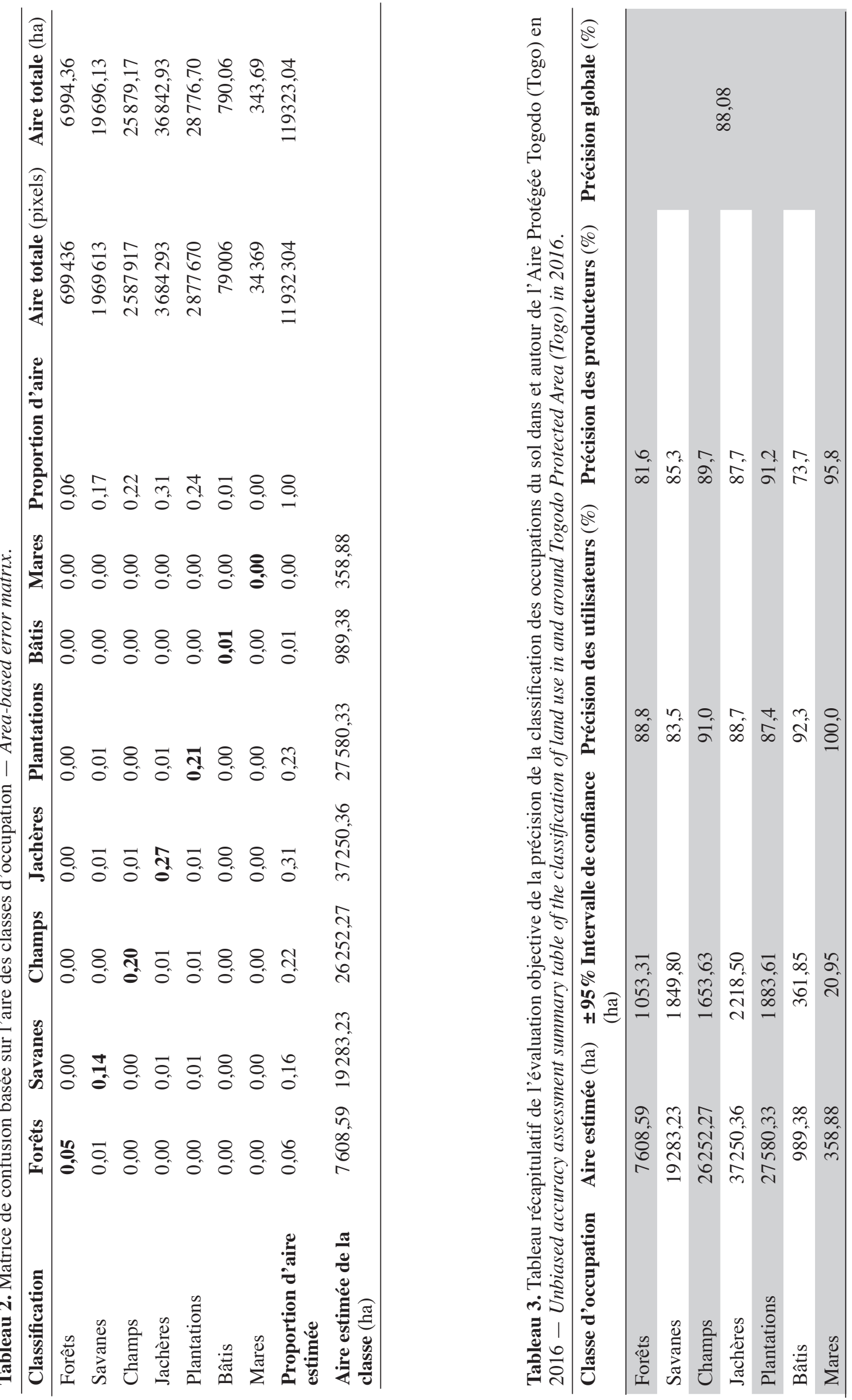


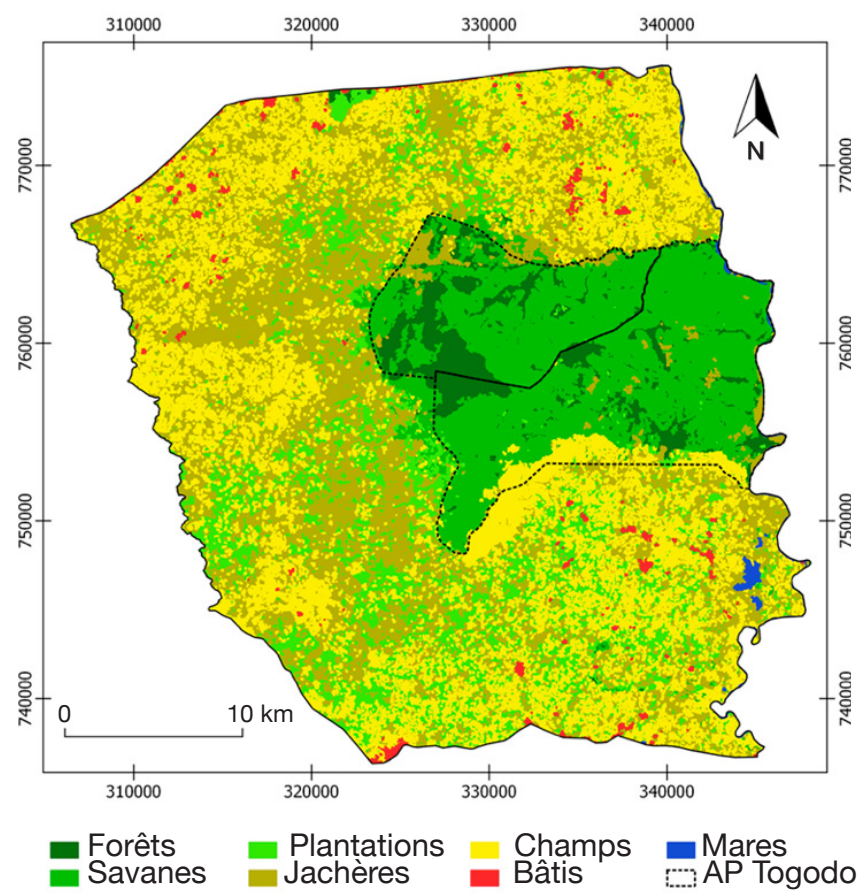

Figure 3. Occupation des terres dans et autour de l'Aire Protégée Togodo (Togo) en 2016 - Land use in and around the Togodo Protected Area (Togo) in 2016.

V.M.Badillo, tandis que celles sur terre ferme sont beaucoup plus dominées par Commelina benghalensis L., Rottboellia cochinchinensis (Lour.) Clayton, Pennisetum polystachion (L.) Schult. et Euphorbia heterophylla $\mathrm{L}$.

Les jachères. Après quelques saisons de cultures, lorsque les sols deviennent pauvres, les champs sont laissés en jachère (flèche noire discontinue) pour favoriser leur restauration. Pendant ce temps, d'autres espaces semi-naturels sont défrichés et mis en valeur. Plusieurs types de jachères présentant une espèce dominante ont été relevés. Les plus fréquentes sont les jachères à Panicum maximum Jacq., à Panicum polystachion L., à Imperata cylindrica (L.) Raeusch., à $C$. odorata et à Tridax procumbens (L.) L. Les jachères à $P$. maximum, à $I$. cylindrica et à $C$. odorata sont très pauvres floristiquement. Les anciennes jachères non remises en culture dans quelques rares cas, par le biais de dynamiques d'enfrichement ou de régénération, évoluent vers des fourrés à Mallotus oppositifolius (Geiseler) Müll.Arg. puis vers des recrûs forestiers (flèche verte).

Les plantations. Les plantations de palmier à huile et de teck sont souvent associées aux cultures selon le système taungya (flèche noire continue). Taungya est un système agroforestier qui implique la plantation d'arbres associée aux cultures agricoles, en particulier les aliments de base de la population locale, et sert ainsi à satisfaire la demande des agriculteurs en terres arables (Kalame et al., 2011). Après quelques saisons de culture, lorsque les plants de teck et de palmier forment une canopée fermée et que les rendements agricoles deviennent faibles, les champs sont abandonnés pour évoluer vers des plantations (teckeraie ou palmeraie). Les teckeraies et les palmeraies, une fois à maturité, sont exploitées partiellement ou totalement (flèche violette) pour laisser place à un sol nu pouvant servir de nouveau de culture. Les plantations, surtout les teckeraies, sont très pauvres floristiquement.

Les éléments paysagers peu perturbés. À l'intérieur de l'aire protégée, le feu de végétation et les vieilles installations illicites de champs sont les principaux facteurs qui influencent la dynamique des formations végétales. La strate herbacée des savanes arbustives, arborées et boisées rencontrées est souvent dominée par P. maximum. Les savanes sont parcourues de façon saisonnière par des feux de végétation (point rouge) accidentels ou planifiés par les forestiers en charge de la gestion de l'aire protégée. En fonction de l'intensité et de la fréquence des feux, on a une évolution progressive des savanes vers les forêts ou inversement (flèches orangées continue et discontinue). Les forêts claires, les forêts sèches semi-décidues et les galeries forestières sont les types de forêts retrouvés.

\subsection{Diversité des plantes envahissantes dominantes}

Au total, 133 relevés floristiques ont été réalisés dont 27 en champs, 41 en jachères, 17 en palmeraies, 13 en teckeraies, 18 en savanes et 17 en forêts. Les 133 relevés floristiques ont permis de recenser 178 espèces dominantes dont 30 envahissantes ou potentiellement envahissantes $(16,85 \%)$. Les plantes envahissantes dominantes se répartissent dans 10 familles botaniques dont 3 sont les plus représentées et regroupent à elles seules $71 \%$ des espèces envahissantes répertoriées : - Poaceae (11 espèces) ;

- Compositae (6 espèces) et Leguminosae ( 5 espèces).

Espèces envahissantes préférentielles. Sur les 30 espèces envahissantes dominantes répertoriées, 2 sont «préférentielles » $(\mathrm{Fr}>20 \%)$. Il s'agit de $P$. maximum et $C$. odorata avec respectivement 49 et 38 occurrences. Parmi les 28 autres espèces envahissantes $(\mathrm{Fr} \leq 20 \%), 9$ sont très rarement présentes et n'ont qu'une seule occurrence (Fr $\leq 0,75 \%)$. C'est le cas d'Acanthospermum hispidum DC., Hyparrhenia hirta (L.) Stapf, Paspalum scrobiculatum L., Pennisetum purpureum Schumach., Ricinus communis L., Senna occidentalis (L.) Link, Sida acuta Burm.f., Synedrella nodiflora (L.) Gaertn. et Typha domingensis Pers. (Figure 5). 


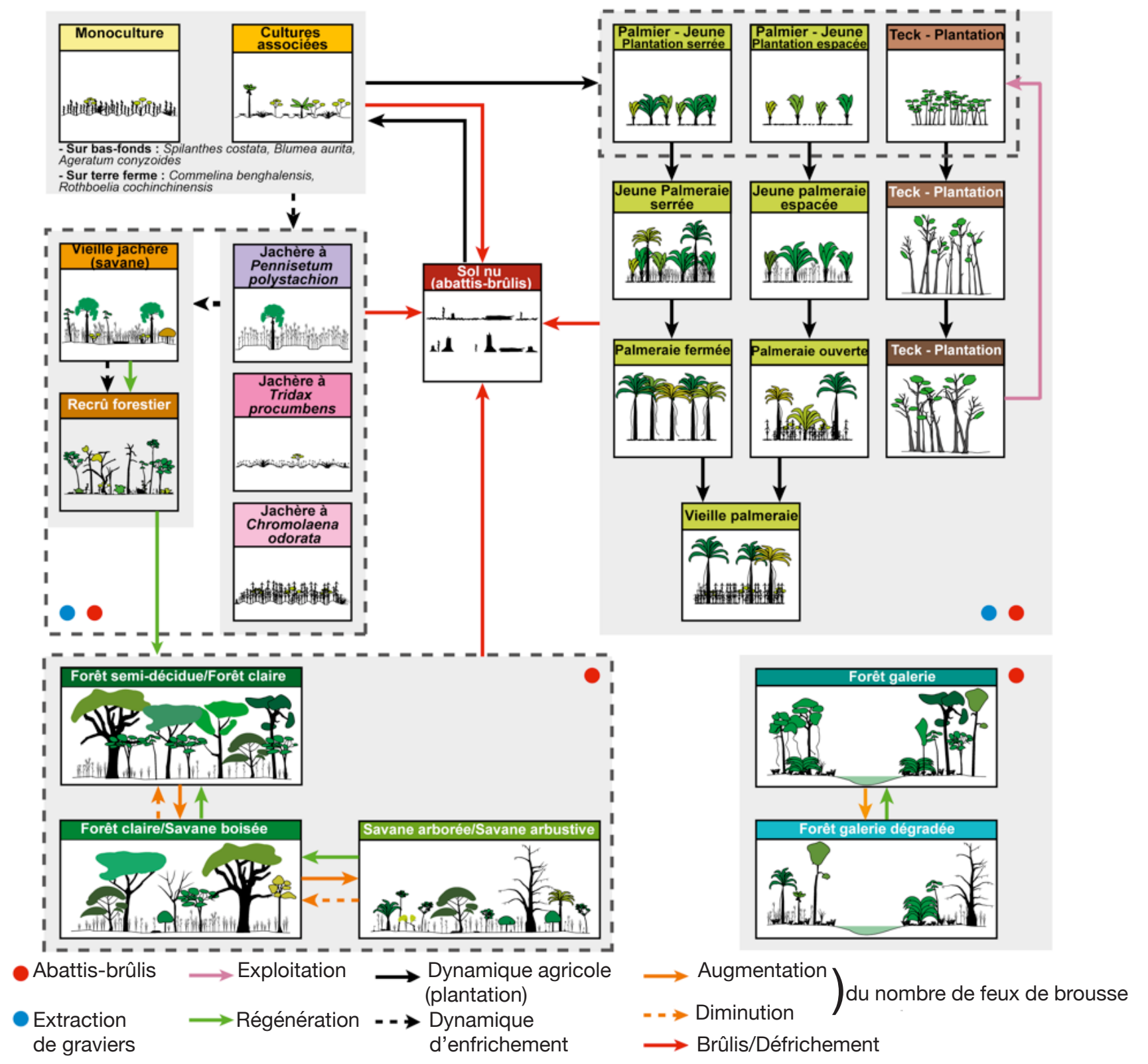

Figure 4. Modèle systémique des dynamiques d'occupation/utilisation du sol au sud-est du Togo en 2016 - Systemic model of land use dynamics in southeastern Togo in 2016.

Les flèches représentent des processus à plus long terme et les ronds représentent des activités ponctuelles - arrows represent long-term processes and rounds represent one-off activities.

Diversité et répartition des plantes envahissantes suivant les différents éléments paysagers. Du point de vue de la diversité, les jachères (25 espèces) et les champs (15 espèces) renferment plus d'espèces envahissantes dominantes que les plantations de teck et les forêts qui ne sont dominées que par quatre et cinq espèces respectivement (Figures 6 et 7). Avec une extrapolation à 50 relevés, les jachères $(28,62 \pm$ $8,55)$ sont significativement plus riches et les forêts $(4,87 \pm 2,93)$ moins riches en plantes envahissantes dominantes que les autres types d'occupation du sol (Tableau 4).

Parmi les espèces envahissantes majeures, $P$. maximum et $C$. odorata sont dominantes dans tous les types d'éléments paysagers, alors que d'autres espèces sont plus dominantes dans un type d'élément paysager particulier. C'est le cas de A. conyzoides, $C$. benghalensis et S. costata, surreprésentées dans les champs et de Triumfetta rhomboidea Jacq., surreprésentée dans les palmeraies (Figure 8). 


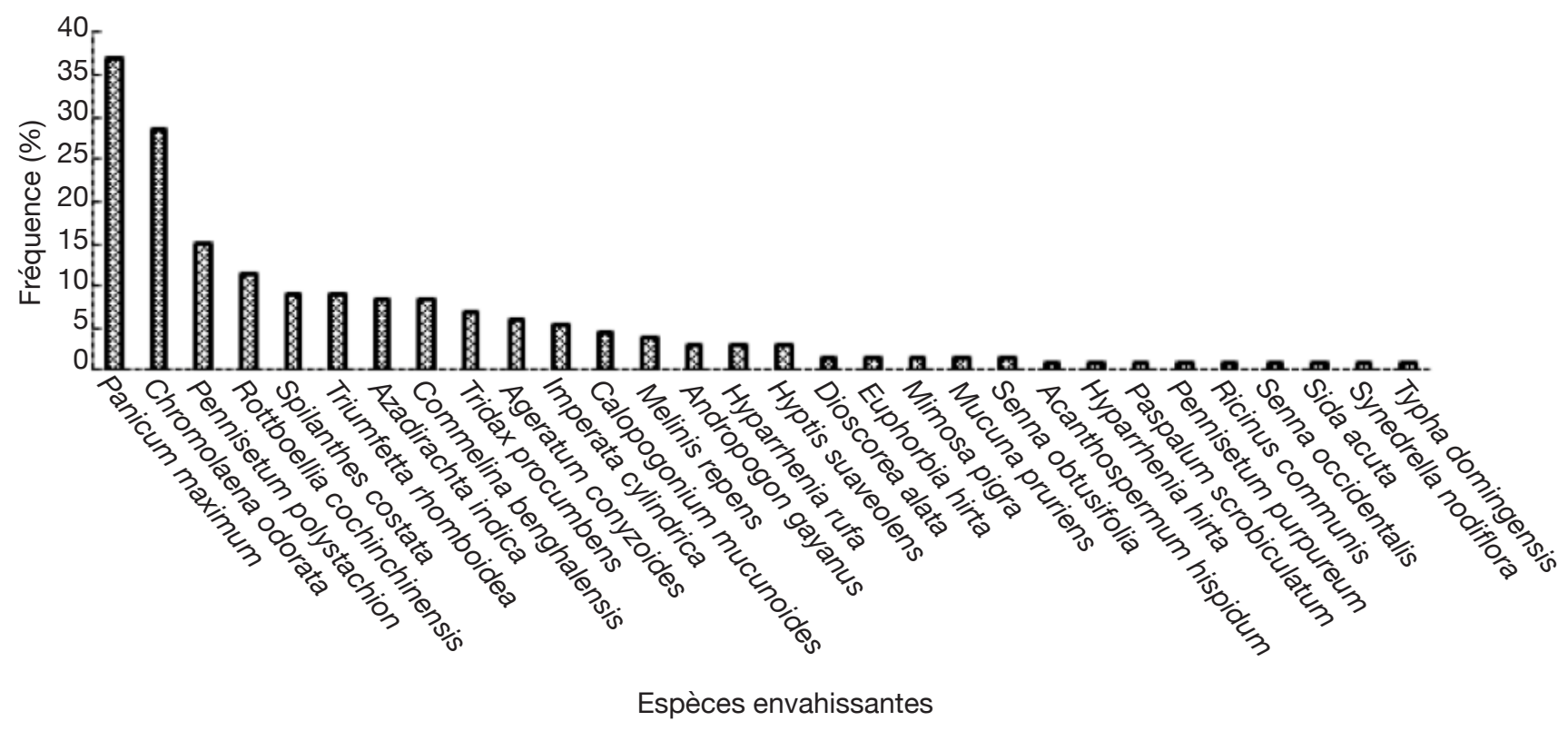

Figure 5. Fréquence d'occurrence des plantes envahissantes dominantes dans et autour de l'Aire Protégée Togodo (Togo) en 2016 - Occurrence frequency of dominant invasive plants in and around the Togodo Protected Area (Togo) in 2016.

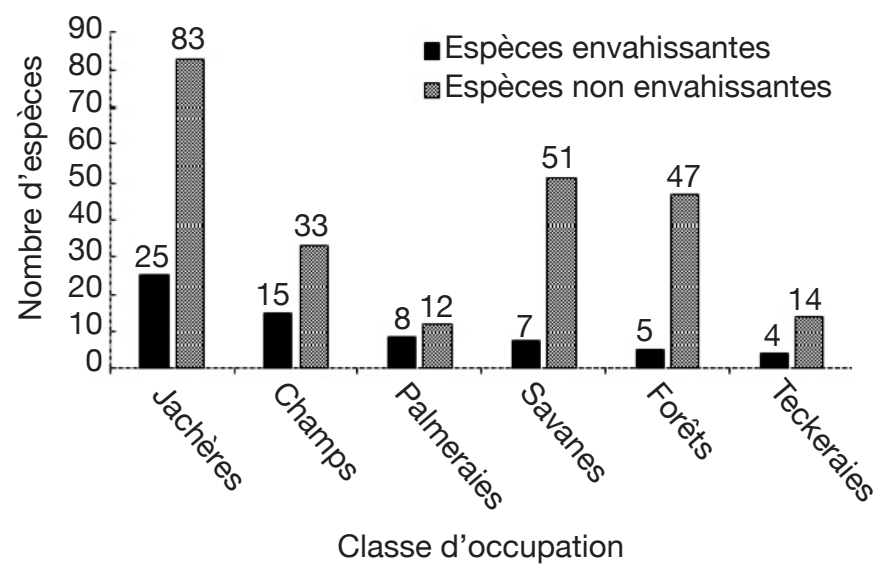

Figure 6. Richesse en plantes envahissantes dominantes des différents types d'occupation/utilisation du sol dans et autour de l'Aire Protégée Togodo (Togo) en 2016 - Richness in dominant invasive plants of different types of land use in and around the Togodo Protected Area (Togo) in 2016.

\section{DISCUSSION}

Cette étude visait à établir un état des lieux sur les dynamiques paysagères en cours dans l'Aire Protégée Togodo et ses environs immédiats, à recenser la diversité et l'abondance des plantes envahissantes dominantes suivant les différents éléments paysagers et à établir une cartographie d'occupation/utilisation des sols pour l'année 2016. Pour l'analyse des dynamiques paysagères, l'approche systémique a été privilégiée. Cette approche basée sur des observations de terrain et permettant d'élaborer un modèle des dynamiques des

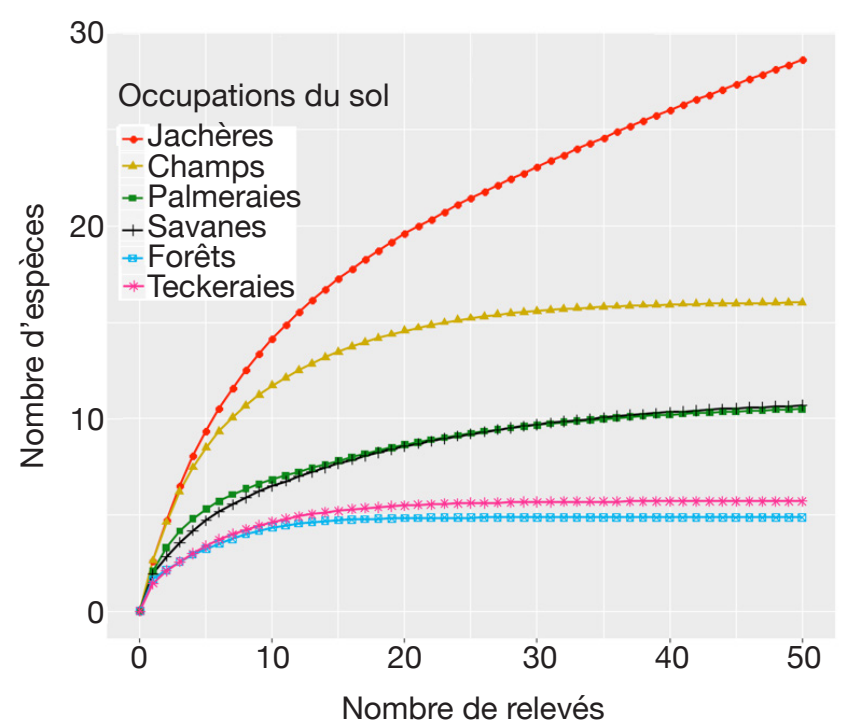

Figure 7. Interpolation (raréfaction) et extrapolation en fonction du nombre de relevés de plantes envahissantes dominantes dans et autour de l'Aire Protégée Togodo au Togo en 2016 - Sample-based interpolation (rarefaction) and extrapolation for surveys of dominant invasive plants in and around the Togodo Protected Area (Togo) in 2016.

éléments paysagers est bien indiquée pour notre site très complexe et en perpétuelle mutation. Elle permet de mieux comprendre les changements en cours et l'évolution probable du milieu si rien n'est fait. Facile à mettre en œuvre, elle facilite aussi l'analyse des effets des utilisations du sol sur les écosystèmes et leurs services. En effet, l'approche systémique a été utilisée 
Tableau 4. Comparaison du nombre d'espèces envahissantes dominantes par occupation du sol extrapolé à 50 relevés dans et autour de l'Aire Protégée Togodo (Togo) en 2016 - Comparison of the number of dominant invasive species by land cover extrapolated to 50 surveys in and around Togodo Protected Area (Togo) in 2016.

\begin{tabular}{|c|c|c|c|c|c|}
\hline $\begin{array}{l}\text { Type d'occupation } \\
\text { du sol }\end{array}$ & $\begin{array}{l}\text { Nombre d'espèces } \\
\text { estimé }\end{array}$ & $\begin{array}{l}95 \% \text { Intervalle de } \\
\text { confiance, limite } \\
\text { inférieure }\end{array}$ & $\begin{array}{l}95 \% \text { Intervalle de } \\
\text { confiance, limite } \\
\text { supérieure }\end{array}$ & Test $X^{2}$ & Résidus du $X^{2}$ \\
\hline Champs & 16,03 & 12,95 & 19,11 & \multirow{6}{*}{$\begin{array}{c}X^{2}=30123 \\
\quad \mathrm{df}=5 \\
p=1395 \mathrm{e}-05\end{array}$} & 0,92 \\
\hline Forêts & 4,87 & 1,94 & 7,81 & & $-2,20$ \\
\hline Jachères & 28,62 & 20,07 & 37,16 & & 4,45 \\
\hline Palmeraies & 10,50 & 4,67 & 16,33 & & $-0,63$ \\
\hline Savanes & 10,68 & 1,07 & 20,30 & & $-0,58$ \\
\hline Teckeraies & 5,71 & $-0,37$ & 11,80 & & $-1,97$ \\
\hline
\end{tabular}

df : nombre de degrés de liberté - number of degrees of freedom.

dans plusieurs études pour l'analyse des dynamiques paysagères mono- ou multi-dates (Oszwald et al., 2007, 2010, 2011).

\subsection{Occupations du sol}

La précision globale (88\%) de notre cartographie est suffisamment élevée pour des analyses d'occupation du sol. Concernant l'organisation spatiale des différents éléments paysagers du milieu, notre cartographie montre que les forêts et les savanes se situent à l'intérieur de l'aire protégée, hormis quelques reliques localisées dans la forêt classée d'Asrama et la forêt sacrée de Godjé-Godjin. Toute la zone autour de l'APT est constituée de champs, de jachères et de plantations. On retrouve également à l'intérieur de l'aire protégée quelques champs et jachère témoignant des activités agricoles et donc des pressions qui menacent les ressources de cette aire. Ces pressions risquent d'augmenter avec la croissance démographique, ce qui constitue un réel défi pour le maintien du statut de conservation de l'APT. Les parcelles agricoles sont contigües à l'aire protégée sans zone tampon. Vu la richesse de ces parcelles en espèces envahissantes, elles constitueraient une source d'introduction continuelle de ces espèces pour l'aire protégée.

\subsection{Dynamiques paysagères gouvernées par des pratiques agricoles}

Notre modèle systémique montre que les pratiques agricoles telles que les défrichements des savanes et forêts pour une mise en culture ou plantation (teck et palmier) et les enfrichements ou mises en jachère sont les principaux facteurs favorisant des dynamiques paysagères. Des résultats similaires ont été obtenus par Kpedenou et al. (2016) dans la préfecture de Yoto où il a été observé une conversion progressive des espaces forestiers en espaces agricoles conduisant à la dégradation des ressources naturelles. Le même constat a été fait au centre-Togo dans le bassin de Mo autour du Parc National Fazao-Malfakassa (Diwediga et al., 2017). De plus, la dynamique des jachères vers les forêts à travers les recrûs forestiers observée dans cette étude a été aussi signalée par Akpagana et al. (1993). Toutefois, dans ces travaux, les différentes formes de conversions anthropiques des composantes du paysage n'ont pas été documentées, comme c'est le cas dans notre étude. La compréhension de ces interdépendances entre les différents éléments paysagers est très informative pour les analyses diachroniques et la simulation des dynamiques futures du milieu, notamment des plantes envahissantes.

\subsection{Les jachères plus dominées par les plantes envahissantes}

Sur les 178 espèces dominantes recensées au cours de notre étude, 30 sont envahissantes ou potentiellement envahissantes, soit $16,85 \%$. Ce taux est largement supérieur aux 4,59\% et 4,60\% obtenus respectivement par Radji et al. (2010b) dans les reliques forestières de la zone écologique V du Togo et par Adjossou (2009) dans les fragments de forêts humides au Togo. Cette différence serait liée, tout d'abord, au fait que ces auteurs n'ont pris en considération que les plantes exotiques et, ensuite, au caractère dominant que nous avons considéré dans le cadre de cette étude. En effet, les plantes envahissantes se caractérisent, entre autres, par un avantage compétitif et une croissance rapide (Valéry et al., 2008), ce qui les rend plus dominantes. Les familles les plus représentées sont les Poaceae, les Compositae et les Leguminosae. Pyšek et al. (2017) ont obtenu des résultats similaires au niveau mondial où les Compositae (1343 espèces), les Poaceae (1 267) et les Leguminosae (1189) contribuent le plus à la flore exotique naturalisée. Parmi les espèces envahissantes dominantes recensées, $P$. maximum et $C$. odorata sont 


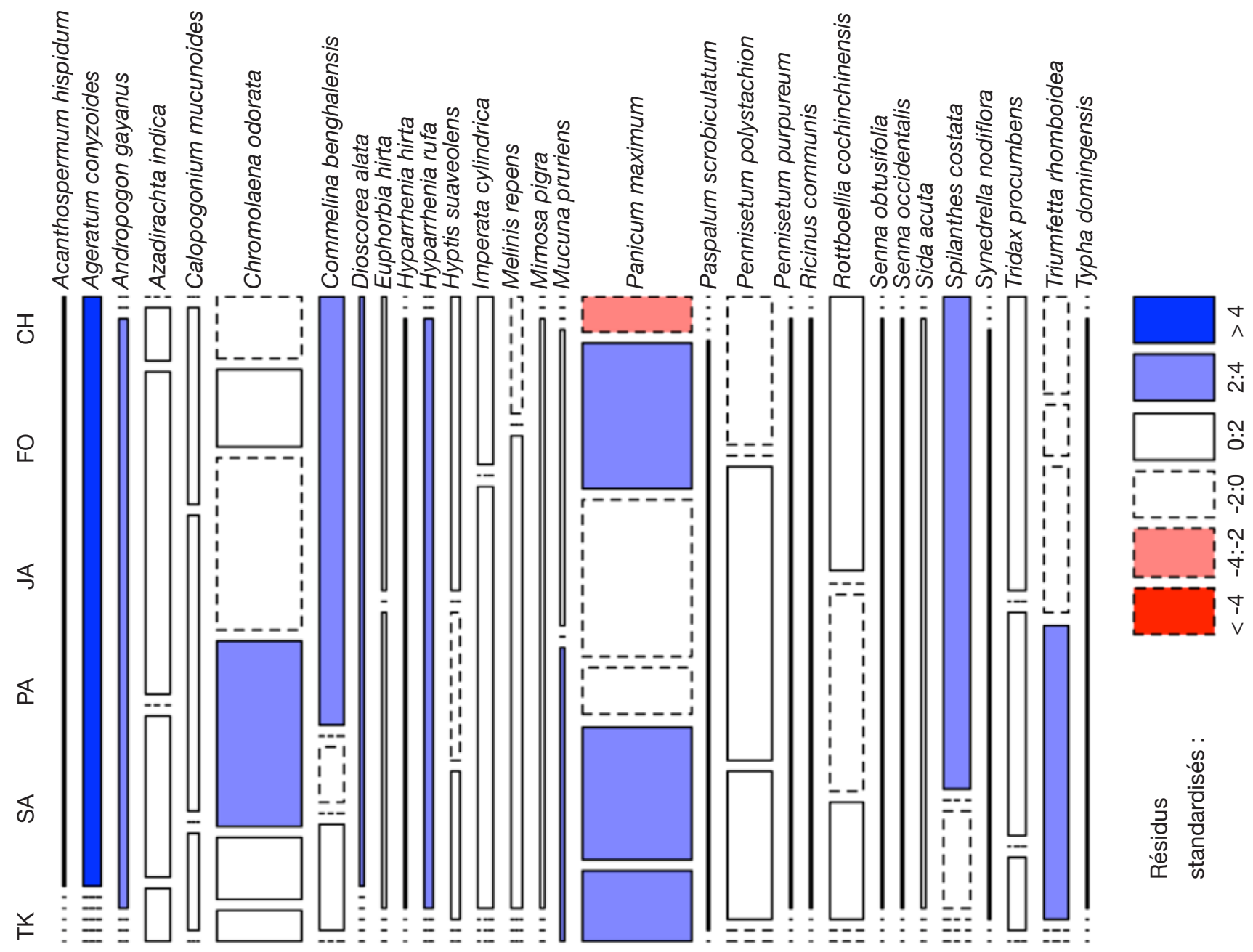

Figure 8. Liaisons entre les espèces de plantes envahissantes dominantes et les utilisations du sol dans et autour de l'Aire Protégée Togodo (Togo) en 2016 - Linkages between dominant invasive plant species and land uses in and around Togodo Protected Area (Togo) in 2016.

$\mathrm{CH}$ : champs - croplands ; FO : forêts - forests ; JA : jachères - fallows ; PA : palmeraies - palm oil plantations ; SA : savanes - savannahs; TK : teckeraies - teak plantations; l'aire de chaque rectangle est proportionnelle à la fréquence relative de la cellule. Plus la bande est large, plus l'espèce concernée est fréquente et plus la bande est longue, plus l'espèce est fréquente dans l'occupation en question. Par exemple, Panicum maximum a une fréquence relative élevée mais est sous-représenté dans les champs (CH). Par contre, Ageratum conyzoides, peu fréquente dans l'ensemble, est surreprésentée dans les champs. Pour les résidus, sous l'hypothèse de l'indépendance, les valeurs correspondent aux probabilités $p<0,05$ et $p<0,0001$, que l'écart par rapport à l'indépendance dépasse 2 ou 4 - the area of each rectangle is proportional to the cell frequency. The larger the cell, the more frequent the species is and the longer the cell, the more frequent the species is in the concerned land use. For example, Panicum maximum has a high frequency but is underrepresented in the croplands $(\mathrm{CH})$. On the other hand, Ageratum conyzoides, generally infrequent, is overrepresented in the croplands. For the standardized deviations, under the assumption of independency, the values correspond to probabilities $\mathrm{p}<0.05$ and $\mathrm{p}<0.0001$ that the absolute value a given standardized deviation is greater than 2 and 4 .

les plus fréquentes et se retrouvent dans tous les types d'éléments paysagers de notre site. Par contre, S. costata est essentiellement rencontrée dans les champs. Spilanthes costata n'étant pas encore signalée au Togo, cette préférence aux champs pourrait s'expliquer par le fait que l'espèce serait introduite récemment et/ou que les pratiques agricoles seraient plus favorables à leurs installation et prolifération. Chromolaena odorata, $P$. maximum et $S$. costata, souvent en groupements denses et pauvres floristiquement, représentent une vraie menace pour l'aire protégée en matière de biodiversité et une corvée pour les agriculteurs lors du désherbage. En effet, P. maximum, bien que ce soit une herbe favorable utilisée comme fourrage dans de nombreuses régions, peut aussi former des peuplements denses et étouffer les espèces indigènes (Global Invasive Species Database, 2018). Aussi, C. odorata est-elle l'une des 100 espèces exotiques envahissantes 
les plus nuisibles dans le monde (Lowe et al., 2000) et son invasion au Togo a été signalée depuis par Akpagana et al. (1993). Concernant la richesse spécifique en plantes envahissantes, les jachères sont les plus envahies et les plantations de teck et les forêts les moins envahies. Cela pourrait se justifier par le degré de perturbation moyen des jachères par rapport aux champs et plantations continuellement perturbés (les labours, l'utilisation des engrais et des pesticides) et par rapport aux savanes et forêts peu perturbées. Car, d'après The Intermediate Disturbance Hypothesis (Eggeling, 1947 ; Connell, 1978) testé et soutenu par plusieurs auteurs (Bongers et al., 2009; Sasaki et al., 2009), la diversité spécifique est plus élevée dans les milieux moyennement perturbés que dans les milieux fortement ou faiblement perturbés. Toutefois, pour mieux soutenir cette hypothèse des effets des perturbations sur les plantes envahissantes sur notre site, il serait utile dans des études futures de prendre en considération d'une part, l'ensemble de la diversité floristique et, d'autre part, les utilisations passées des terres qui constituent un facteur d'explication des invasions par les plantes (Vilà \& Ibáñez, 2011). L'idéal serait de réaliser les observations de terrain de ces études sur une année complète afin d'inclure toutes les saisons.

\section{CONCLUSIONS ET PERSPECTIVES}

La compréhension de l'effet des activités anthropiques sur l'abondance et la distribution des plantes envahissantes est une nécessité dans le contexte actuel des changements globaux. Dans notre étude réalisée dans et autour de l'Aire Protégée Togodo, les différents types d'utilisation et de conversion anthropiques des éléments paysagers ont été documentés, de même que la diversité en plantes envahissantes dominantes. Nos résultats montrent que les pratiques agricoles, en l'occurrence l'agriculture itinérante sur brûlis, la mise en jachère et les plantations de teck et palmier à huile sont les principales causes des dynamiques paysagères dans le milieu. Le paysage de notre site est très diversifié en plantes envahissantes $(16,85 \%)$. Les jachères sont les plus dominées par les plantes envahissantes. Les espèces comme $P$. maximum, $C$. odorata et $S$. costata représentent un réel défi aussi bien pour l'aire protégée que pour les agriculteurs. Cette étude constitue une base idéale pouvant servir à l'élaboration de stratégie de gestion des plantes envahissantes, mais aussi pour des recherches sur l'effet des utilisations passées des terres sur les plantes envahissantes dans et autour de l'APT. Il ressort de cette étude que l'analyse systémique paysagère et la télédétection constituent des outils opérationnels pour une meilleure compréhension de la problématique des plantes envahissantes dans les écosystèmes anthropisés des savanes et forêts sèches de l'Afrique de l'Ouest. Ces écosystèmes, très peu étudiés pour leur invasion végétale, sont pourtant très riches en plantes envahissantes et méritent donc plus d'attention, surtout au niveau des aires protégées.

\section{Remerciements}

Les auteurs remercient la Banque Islamique de Développement (BID), qui a soutenu cette recherche à travers son Programme de Bourses de Mérite. Les auteurs voudraient remercier aussi Dr Wouyo Atakpama et le Laboratoire de Botanique et Écologie Végétale (LBEV) pour leurs apports dans la collecte des données et la détermination des espèces.

\section{Bibliographie}

Aboh B.A., Houinato M., Oumorou M. \& Sinsin B., 2008. Capacités envahissantes de deux espèces exotiques, Chromolaena odorata (Asteraceae) et Hyptis suaveolens (Lamiaceae), en relation avec l'exploitation des terres de la région de Bétécoucou (Bénin). Belg. J. Bot., 141(2), 125-140.

Adjossou K., 2009. Diversité, structure et dynamique de la végétation dans les fragments de forêts humides du Togo : les enjeux pour la conservation de la biodiversité. Thèse de doctorat : Université de Lomé (Togo).

Akpagana K., Guelly K.A. \& Gumedzoe Y.M., 1993. Une adventice en voie d'envahissement du territoire togolais : Eupatorium odoratum L. [syn. Chromolaena odorata (L.) R.M. King \& Robinson] (Compositae). Acta Bot. Gallica, 140(5), 535-543.

Baatz M. \& Schäpe A., 2000. Multiresolution segmentation: an optimization approach for high quality multiscale image segmentation. In: Strobl J., Blaschke T. \& Griesebner G., eds. Angewandte Geographische Informations-Verarbeitung XII. Karlsruhe, Germany: Wichmann-Verlag, 12-23.

Bellard C. \& Jeschke J.M., 2016. A spatial mismatch between invader impacts and research publications. Conserv. Biol., 30(1), 230-232.

Benz U.C. et al., 2004. Multi-resolution, object-oriented fuzzy analysis of remote sensing data for GIS-ready information. ISPRS J. Photogramm. Remote Sens., 58(34), 239-258.

Bongers F., Poorter L., Hawthorne W.D. \& Sheil D., 2009. The intermediate disturbance hypothesis applies to tropical forests, but disturbance contributes little to tree diversity. Ecol. Lett., 12(8), 798-805.

Braun-Blanquet J., Roussine N. \& Nègre R., 1952. Les groupements végétaux de la France méditerranéenne. Montpellier, France : CNRS, 292.

Burel F. \& Baudry J., 1999. Écologie du paysage. Concepts, méthode et applications. Paris : Lavoisier. 
Burgess N. et al., 2004. Terrestrial ecoregions of Africa and Madagascar: a conservation assessment. Washington, DC: Island Press.

CABI, 2017. Invasive species compendium. Wallingford, UK: CAB International.

Chatelain C., Gautier L. \& Spichiger R., 1996. A recent history of forest fragmentation in southwestern Ivory Coast. Biodivers. Conserv., 5(1), 37-53.

CILSS, 2016. Landscapes of West Africa: a window on a changing world. Ouagadougou: CILSS.

Clavel J., Julliard R. \& Devictor V., 2011. Worldwide decline of specialist species: toward a global functional homogenization? Front. Ecol. Environ., 9(4), 222-228.

Colwell R.K. et al., 2012. Models and estimators linking individual-based and sample-based rarefaction, extrapolation and comparison of assemblages. J. Plant Ecol., 5(1), 3-21.

Connell J.H., 1978. Diversity in tropical rain forests and coral reefs. Science, 199(4335), 1302-1310.

Dessaint F., Chadoeuf R. \& Barralis G., 2001. Diversité des communautés de mauvaises herbes des cultures annuelles de Côte d'Or (France). Biotechnol. Agron. Soc. Environ., 5(2), 91-98.

DGSCN, 2010. Recensement général de la population et de l'habitat au Togo. Résultats définitifs. Lomé : DGSCN.

Diwediga B., Agodzo S., Wala K. \& Le Q.B., 2017. Assessment of multifunctional landscapes dynamics in the mountainous basin of the Mo River (Togo, West Africa). J. Geogr. Sci., 27(5), 579-605.

Eggeling W.J., 1947. Observations on the ecology of the Budongo rain forest, Uganda. J. Ecol., 34, 20-87.

Elton C.S., 1958. The ecology of invasions by animals and plants. Chicago, Il, USA: The University of Chicago Press.

Ern H., 1979. Die Vegetation Togos. Gliederung, Gefährdung, Erhaltung. Willdenowia, 9, 295-312.

Fousseni F. et al., 2012. Assessment and impact of anthropogenic disturbances in protected areas of northern Togo. For. Stud. China, 14(3), 216-223.

Foxcroft L.C., Richardson D.M., Rejmánek M. \& Pyšek P., 2010. Alien plant invasions in tropical and sub-tropical savannas: patterns, processes and prospects. Biol. Invasions, 12(12), 3913-3933.

Friendly M., 1994. Mosaic displays for multi-way contingency tables. J. Am. Stat. Assoc., 89(425), 190200.

Global Invasive Species Database, 2018. Species profile: Urochloa maxima, http://www.iucngisd.org/gisd/ species.php?sc=398, (27/02/2018).

Holou R.A., Achigan-Dako E.G. \& Sinsin B., 2013. Ecology and management of invasive plants in Africa. In: Invasive plant ecology. Boca Raton, FL, USA: CRC Press, 161-174.

Kalame F.B. et al., 2011. Modified taungya system in Ghana: a win-win practice for forestry and adaptation to climate change? Environ. Sci. Policy, 14(5), 519-530.
Kpedenou K.D., Boukpessi T. \& Tchamie T.T.K., 2016. Quantification des changements de l'occupation du sol dans la préfecture de Yoto (sud-est Togo) à l'aide de l'imagerie satellitaire Landsat. Rev. Sci. Environ., 13, 137-156.

Latombe G. et al., 2017. A vision for global monitoring of biological invasions. Biol. Conserv., 213, 295-308.

Leung B. et al., 2002. An ounce of prevention or a pound of cure: bioeconomic risk analysis of invasive species. Proc. R. Soc. London, Ser. B, 269(1508), 2407-2413.

Levine J.M. et al., 2003. Mechanisms underlying the impacts of exotic plant invasions. Proc. R. Soc. London, Ser. B, 270(1517), 775-781.

Lowe S., Browne M., Boudjelas S. \& De Poorter M., 2000. 100 of the world's worst invasive alien species. A selection from the global invasive species database. Auckland, New Zealand: The Invasive Species Specialist Group (ISSG).

Maxwell S.L., Fuller R.A., Brooks T.M. \& Watson J.E., 2016. Biodiversity: the ravages of guns, nets and bulldozers. Nature, 536(7615), 143.

Olofsson P., Foody G.M., Stehman S.V. \& Woodcock C.E., 2013. Making better use of accuracy data in land change studies: estimating accuracy and area and quantifying uncertainty using stratified estimation. Remote Sens. Environ., 129, 122-131.

Oszwald J., Atta J.-M.K., Kergomard C. \& Robin M., 2007. Représenter l'espace pour structurer le temps : approche des dynamiques de changements forestiers dans le sudest de la Côte d'Ivoire par télédétection. Télédétection, 7(1-2-3-4), 271-282.

Oszwald J. et al., 2010. Analyse des directions de changement des états de surface végétaux pour renseigner la dynamique du front pionnier de Maçaranduba (Brésil) entre 1997 et 2006. Télédétection, 9(2), 97-111.

Oszwald J. et al., 2011. Identification d'indicateurs de changement d'occupation du sol pour le suivi des mosaïques paysagères. Bois For. Trop., 307(1), 721.

Paini D.R. et al., 2016. Global threat to agriculture from invasive species. Proc. Natl. Acad. Sci. U.S.A., 113(27), 7575-7579.

Pimentel D., 2002. Biological invasions: economic and environmental costs of alien plant, animal, and microbe species. Boca Raton, Fl, USA: CRC Press.

Pontius R.G. \& Millones M., 2011. Death to Kappa: birth of quantity disagreement and allocation disagreement for accuracy assessment. Int. J. Remote Sens., 32(15), 4407-4429.

Pratt C.F., Constantine K.L. \& Murphy S.T., 2017. Economic impacts of invasive alien species on African smallholder livelihoods. Global Food Secur., 14, 31-37.

Pyšek P. et al., 2010. Disentangling the role of environmental and human pressures on biological invasions across Europe. Proc. Natl. Acad. Sci. U.S.A., 107(27), 1215712162. 
Pyšek P. et al., 2017. Naturalized alien flora of the world: species diversity, taxonomic and phylogenetic patterns, geographic distribution and global hotspots of plant invasion. Preslia, 89(3), 203-274.

Radji R., Kokou K. \& Akpagana K., 2010a. Étude diagnostique de la flore ornementale togolaise. Int. J. Biol. Chem. Sci., 4(2), 491-508.

Radji R., Klu K. \& Kokou K., 2010b. Forest invasion by alien plant species: the case of neem tree (Azadirachta indica A.Juss.) in Southern Togo. Int. J. Biodivers. Conserv., 2(10), 300-307.

Robin M., 1998. La télédétection. Paris : Nathan Université.

Sala O.E. et al., 2000. Global biodiversity scenarios for the year 2100. Science, 287(5459), 1770-1774.

Santos F., 2018. Le kappa de Cohen : un outil de mesure de l'accord inter-juges sur des caractères qualitatifs, http:// www.pacea.u-bordeaux 1.fr/IMG/pdf/Kappa_Cohen.pdf $1-5,(17 / 01 / 2019)$.

Sasaki T. et al., 2009. Management applicability of the intermediate disturbance hypothesis across Mongolian rangeland ecosystems. Ecol. Appl., 19(2), 423-432.

Ségniagbéto G.H. et al., 2018. Local distribution and density estimates of primates in the Transboundary Reserve of the Mono River, Togo (West Africa). Rev. Ecol. (Terre et Vie), 73(3), 363-374.

Sparfel L., Gourmelon F. \& Le Berre I., 2008. Approche orientée-objet de l'occupation des sols en zone côtière. Rev. Télédétection, 8(4), 237-256.
Tassin J., 2015. Les plantes invasives : un ajustement du vivant à notre monde. CIHEAM Watch Lett., 33, 99-103.

Tassin J., Sarrailh J.-M. \& Rivière J.-N., 2007. Essences forestières et invasions: des systèmes de prédiction toujours plus fiables. Bois For. Trop., 292, 71-79.

The Plant List, 2013. The plant list. Version 1.1., http:// www.theplantlist.org/. http://www.theplantlist.org/, (10/02/2017).

UICN, 2017. Global invasive species database, http://www. iucngisd.org/gisd/search.php, (10/02/2017).

UICN/PACO, 2013. Plantes invasives affectant les aires protégées d'Afrique de l'Ouest : gestion pour la réduction des risques pour la biodiversité. Ouagadougou: UICN/ PACO.

Valéry L., Fritz H., Lefeuvre J.-C. \& Simberloff D., 2008. In search of a real definition of the biological invasion phenomenon itself. Biol. Invasions, 10(8), 1345-1351.

Vilà M. et al., 2011. Ecological impacts of invasive alien plants: a meta-analysis of their effects on species, communities and ecosystems. Ecol. Lett., 14(7), 702708.

Vilà M. \& Ibáñez I., 2011. Plant invasions in the landscape. Landscape Ecol., 26(4), 461-472.

Weber E., 2003. Invasive plant species of the world: a reference guide to environmental weeds. CABI.

(61 réf.) 